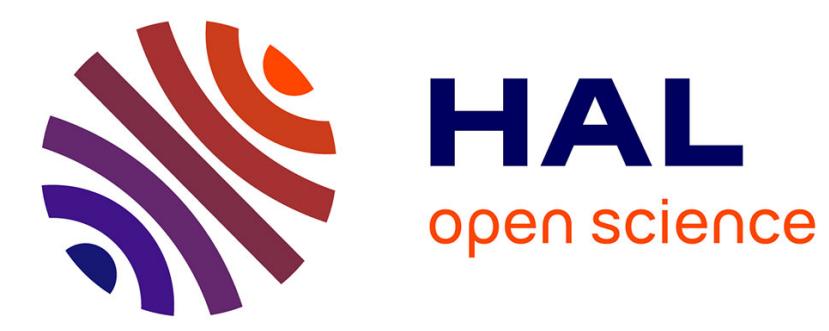

\title{
Investigating the influence of local porosity variations and anisotropy effects on the permeability of fibrous media for air filtration
}

Sonia Woudberg, Félicie Theron, Elisabeth Lys, Laurence Le Coq

\section{- To cite this version:}

Sonia Woudberg, Félicie Theron, Elisabeth Lys, Laurence Le Coq. Investigating the influence of local porosity variations and anisotropy effects on the permeability of fibrous media for air filtration. Chemical Engineering Science, 2018, 180, pp.70 - 78. 10.1016/J.CES.2018.01.035 . hal-01700159

\author{
HAL Id: hal-01700159 \\ https://hal.science/hal-01700159
}

Submitted on 21 Aug 2019

HAL is a multi-disciplinary open access archive for the deposit and dissemination of scientific research documents, whether they are published or not. The documents may come from teaching and research institutions in France or abroad, or from public or private research centers.
L'archive ouverte pluridisciplinaire HAL, est destinée au dépôt et à la diffusion de documents scientifiques de niveau recherche, publiés ou non, émanant des établissements d'enseignement et de recherche français ou étrangers, des laboratoires publics ou privés. 


\title{
Investigating the influence of local porosity variations and anisotropy effects on the permeability of fibrous media for air filtration
}

\author{
Sonia Woudberg ${ }^{\mathrm{a}, *}$, Félicie Theron ${ }^{\mathrm{b}}$, Elisabeth Lys $^{\mathrm{c}}$, Laurence Le Coq $^{\mathrm{b}}$ \\ ${ }^{a}$ Applied Mathematics, Department of Mathematical Sciences, Stellenbosch University, Private Bag X1, Matieland 7602, South Africa \\ ${ }^{\mathrm{b}}$ IMT Atlantique, GEPEA, CNRS, CS 20722, 44307 Nantes, France \\ ' IMT Atlantique, SUBATECH, CNRS, CS 20722, 44307 Nantes, France
}

Keywords:

Fibrous medium

Porous structure

Permeability

Modelling

\section{A B S T R A C T}

The permeability prediction of real fibrous media remains a major challenge due to their complex porous structure. The existing models generally take two parameters into account that depict the average structural properties of fibrous media: porosity (or solid volume fraction) and fibre diameter (or radius). Due to the local variations that those parameters can exhibit the models often fail to accurately predict the permeability. In this study experimental data of three fibrous media of different porous structures employed in air filtration are used to assess numerous empirical and analytical models from the literature. It is found that all the models considered significantly under-predict the experimental permeability values. Special attention is given to the analytical Representative Unit Cell (RUC) model which is physically adaptable and can therefore be used to investigate the aforementioned discrepancies between model and experiment. Local porosity variations are investigated through a sensitivity analysis on the minimum and maximum solid volume fraction values. Incorporation of the porosity variations into the RUC model significantly reduces its under-prediction and may also serve as explanation for the discrepancies observed. An adaptation to the RUC model is proposed that takes into account the anisotropy effects as a result of the stacking of fibrous medium sheets into several layers and the increase in porosity as a result thereof. The adapted analytical model gives an indication on the factor to apply on the mean porosity in order to incorporate the anisotropy effect into the model prediction, and so reduce the observed under-predictions.

\section{Introduction}

The permeability of fibrous porous media depends on the medium's structural properties: fibre thickness, morphology, size 


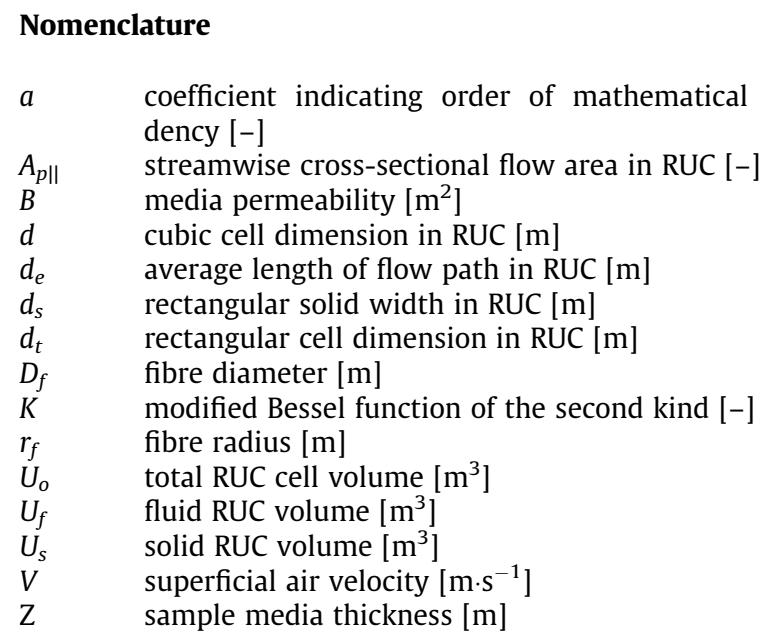

$\begin{array}{ll}\text { Greek letters } \\ \alpha & \text { coefficient in Tomadakis and Robertson model [-] } \\ \gamma & \text { factor by which the cell dimension } d \text { is increased [-] } \\ \delta & \text { fraction of fluid flowing through local porosity region [- } \\ \varepsilon & \text { porosity [-] } \\ \mu & \text { air dynamic viscosity [Pa.s] } \\ \phi & \text { solid volume fraction [-] } \\ \Delta P & \text { pressure drop [-] } \\ \text { Subscripts } \\ \text { adapt } & \text { adapted } \\ \text { exp } & \text { experimental } \\ \text { iso } & \text { isotropic } \\ \text { max } & \text { maximum } \\ \text { min } & \text { minimum } \\ \text { mod } & \text { modelled } \\ \text { sens } & \text { sensitivity } \\ \text { TP } & \text { through plane }\end{array}$

distribution, diameter to length ratio and arrangement. The determination of the permeability of fibrous media for aerosol filtration (in specific) is an important aspect as this parameter influences the filter performances (i.e. pressure drop and collection efficiency). The earlier attempts in predicting the permeability of fibrous porous media were based on the assumption of isotropy. The permeability prediction assuming isotropy, i.e. $B_{i s o}$, of Jackson and James (1986) and Davies (1952), for example, are based on empirical models and respectively given by:

$B_{\text {iso }}=\frac{D_{f}^{2}}{4} \frac{3}{20 \phi}(-\ln \phi-0.931)$

and

$B_{\text {iso }}=\frac{D_{f}^{2}}{4\left[16 \phi^{3 / 2}\left(1+56 \phi^{3}\right)\right]}$,

where $D_{f}$ is the fibre diameter and $\phi$ denotes the solid volume fraction. The implicit analytical model of Spielman and Goren (1968) also based on isotropic media, is given by:

$\frac{1}{4 \phi}=\frac{1}{3}+\frac{5}{6} \frac{\sqrt{B_{\text {iso }}}}{r_{f}} \frac{K_{1}\left(\frac{r_{f}}{\sqrt{B_{\text {iso }}}}\right)}{K_{0}\left(\frac{r_{f}}{\sqrt{B_{\text {iso }}}}\right)}$,

where $K_{0}$ and $K_{1}$ are the modified Bessel functions of the second kind and $r_{f}=D_{f} / 2$ denotes the fibre radius. More recently Tomadakis and Robertson (2005) proposed the following permeability prediction for isotropic media:

$B_{\text {iso }}=\frac{(1-\phi) D_{f}^{2}}{32 \ln ^{2}(1-\phi)} \frac{\left(1-\phi-\varepsilon_{p}\right)^{\alpha+2}}{\left(1-\varepsilon_{p}\right)^{\alpha}\left[(\alpha+1)(1-\phi)-\varepsilon_{p}\right]^{2}}$,

with $\varepsilon_{p}=0.037$ a percolation threshold porosity and $\alpha=0.661$.

Due to their manufacturing processes, fibrous media can, however, exhibit anisotropic fibrous structures (e.g. Theron et al., 2017). Moreover, due to their implementation in ventilation facilities, the airflow mostly penetrates the filter through pathlines normal to the medium. Therefore, for this kind of medium the permeability is generally determined through pressure drop measurements in flat configurations normal to the flow, known as the through plane permeability. Analytical permeability predictions for through plane (and in-plane) flow were proposed for anisotropic media by e.g. Spielman and Goren (1968) Tomadakis and Robertson (2005), Tamayol and Bahrami (2010) and Van Doormal and Pharoah (2009). The following implicit analytical equation for the through plane permeability prediction $\left(B_{T P}\right)$ was provided by Spielman and Goren (1968):

$\frac{1}{4 \phi}=\frac{1}{2}+\frac{\sqrt{B_{T P}}}{r_{f}} \frac{K_{1}\left(\frac{r_{f}}{\sqrt{B_{T P}}}\right)}{K_{0}\left(\frac{r_{f}}{\sqrt{B_{T P}}}\right)}$.

The permeability prediction for through plane flow provided by Tomadakis and Robertson (2005) is given by the following Tomadakis and Robertson through plane model:

$B_{T P}=\frac{(1-\phi) D_{f}^{2}}{32 \ln ^{2}(1-\phi)} \frac{\left(1-\phi-\varepsilon_{p}\right)^{\alpha+2}}{\left(1-\varepsilon_{p}\right)^{\alpha}\left[(\alpha+1)(1-\phi)-\varepsilon_{p}\right]^{2}}$.

with $\varepsilon_{p}=0.11$ and $\alpha=0.785$.

Tamayol and Bahrami (2011) have classified the through plane and in-plane permeability models as representative of that of $2 \mathrm{D}$ fibrous media. In such media all the fibres lie in planes that are parallel to each other, but may take on random orientations, albeit still classified as anisotropic because of the 2D configuration. Several models have also been proposed in the literature for flow through 1D fibrous media (Woudberg, 2017) in which the fibres also lie in parallel planes but with their axes directed parallel with respect to each other. In such a case the flow is regarded as parallel or perpendicular (or transverse) to the fibre axes and anisotropic. The first analytical models for flow parallel and perpendicular to 1D fibrous media were proposed by e.g. Happel (1959), Drummond and Tahir (1984) and Gebart (1992). Model predictions by Tomadakis and Robertson (2005) and Tamayol and Bahrami (2010) followed in more recent years.

The isotropic permeability models mentioned above fall in the category of 3D fibrous media. An alternative analytical approach in producing $3 \mathrm{D}$ isotropic permeability models, apart from the models of Spielman and Goren (1968) and Tomadakis and Robertson (2005), discussed above, is by allocating a weight of $1 / 3$ to the permeability prediction for flow parallel to $1 \mathrm{D}$ fibrous 
media and a weight of $2 / 3$ to that for flow perpendicular to $1 \mathrm{D}$ fibrous media. This concept was initially introduced by Jackson and James (1986) and later by Mattern and Deen (2008) as a mixing rule. Clague and Phillips (1997) have applied the weighted average approach to the permeability prediction for flow parallel to 1D fibres proposed by Drummond and Tahir (1984) and the permeability prediction for flow perpendicular to 1D fibres of Sangani and Acrivos (1982). Woudberg (2017) proposed additional isotropic (abbreviated, iso) models based on the weighted average approach. One is based on the parallel and transverse flow models of Happel (1959), yielding the following Happel iso model:

$B_{\text {iso }}=\frac{D_{f}^{2}}{4}\left[\frac{1}{3} 4 \phi\left(-\ln \phi-\frac{3}{2}+2 \phi-\frac{\phi^{2}}{2}\right)^{-1}+\frac{2}{3} 8 \phi\left(-\ln \phi+\frac{\phi^{2}-1}{\phi^{2}+1}\right)^{-1}\right]^{-1}$,

and the other on the parallel and transverse flow models of Tomadakis and Robertson (2005), yielding the following Tomadakis and Robertson iso model:

$B_{\text {iso }}=\frac{D_{f}^{2}}{4}\left[\frac{1}{3}\left(\frac{(1-\phi)^{3}}{8 \phi^{2}}\right)^{-1}+\frac{2}{3}\left(\frac{(1-\phi)^{3}}{8 \phi^{2}} \frac{\exp (-\alpha \phi)}{(1+\alpha(1-\phi))^{2}}\right)^{-1}\right]^{-1}$,

with $\alpha=1.16$. Eq. (8) will be referred to as the Tomadakis and Robertson iso alternative (abbreviated, iso alt) model.

The permeability prediction of real fibrous media remains challenging, despite the numerous attempts in the literature mentioned above. All the models presented above are functions of the average fibre diameter and solid volume fraction. These structural parameters can, however, exhibit significant local deviations. Moreover there are only a few studies in the literature (e.g. Jaganathan et al., 2008; Gervais et al., 2012; Huang et al., 2015) that present actual data (such as permeability, fibre diameter and porosity or solid volume fraction) that enables one to assess the validity of the existing models. Recently Soltani et al. (2017) used $\mathrm{X}$-ray micro-computed tomography and simulations to generate realistic and virtual 3D fibrous structures of porosity ranging from 0.74 to 0.93 . On the basis of the obtained numerical domains they predicted both through-plane and in-plane permeabilities by fluid flow simulations. These numerical results were then compared to experimental data as well as available empirical, analytical, numerical and experimental results in terms of dimensionless permeability, i.e. permeabilities divided by the square of fibre radius.

The objectives of this study are to compare experimental permeability values for airflow through fibrous media to model predictions from the literature and to investigate the extent of local porosity variations and increase in porosity as a result of the stacking of medium sheets (yielding an additional anisotropy) on the permeability prediction. The latter effect will henceforth be referred to as the stacking effect. The fibrous media examined experimentally may fall in the category of 2D fibrous media because of the random orientation of the fibres exhibited in planes parallel to each other. In addition, only through plane flow were considered and the structural parameters have been characterized with as much accuracy as possible. As a result, the 2D anisotropic through plane models will be assessed in this study and henceforth be referred to as the through plane (TP) models as opposed to the isotropic models. The isotropic models will also be assessed to serve as reference for the TP models. In Section 2 the existing analytical Representative Unit Cell (RUC) model of Woudberg (2017) for through plane flow and isotropic fibrous media will be discussed.

The structural properties and permeabilities of the three real fibrous media used to assess these models as well as those consid- ered from the literature are detailed in Section 3. In Section 4 the model comparison is presented and discussed. Special attention is paid to the sensitivity of models to the structural properties since their experimental characterization can lead to significant deviations. As a result, a sensitivity analysis is performed on the isotropic RUC model in order to investigate the influence of local in-plane porosity variations on the permeability prediction. The objective of Section 5 is to adapt the isotropic RUC model in order to estimate the increase in porosity as a result of the stacking effect.

\section{RUC models}

In order to depict the experimental permeability generally obtained by permeametry for anisotropic fibrous media, Woudberg (2017) presented a 2D geometrical model consisting of rectangular fibres in a cubic Representative Unit Cell, known as the RUC model, and schematically shown on the left hand side in Fig. 1. The two solid fibres orientated in the two principal 2D directions of a rectangular Cartesian coordinate system, represent the average geometry of the actual fibres that have random orientations in planes parallel to each other. The surfaces of the average fibre geometry adjacent to the RUC in the through plane flow direction is also indicated. Note that the second solid fibre layer is not included in the RUC, although the effect of the adjacent layer is indeed taken into consideration to establish the piece-wise parallel plate geometry between which the flow takes place in the limit of low Reynolds number flow. Only the through plane flow direction is indicated (by the arrow) since the in-plane direction was not considered in the experiments of which the data will be used to assess the model. The through plane flow direction corresponds to the streamwise direction. The isotropic RUC model is shown on the right hand side of Fig. 1.

The isotropic RUC model results from placing three of the solid fibres in the 1D fibre RUC model (refer to Fig. 1 of Woudberg, 2017) in the same cubic RUC such that the fibres are orthogonal with respect to each other and two of the fibres are directed perpendicular to the flow direction and the other one parallel.

The rectangular fibre orientated in each of the three principal directions resembles the extent of solid contribution in each direction as a result of the 3D randomness of actual fibrous media. This model will be used as reference in addition to the through plane model to assess the experimental permeability data.

In Fig. 1 the cubic cell dimension is denoted by $d$. There are different ways in which to convert the rectangular fibre geometry used in the RUC model to cylindrical geometry in order to find an estimation for a representative fibre diameter. The approach followed by Woudberg (2017) was to set the cross-sectional area of a rectangular and cylindrical fibre equal, i.e. $d_{s}^{2}=\pi r_{f}^{2}$, with $d_{s}$ being the rectangular solid width of the fibre in the RUC model, as indicated in Fig. 1. In this study an alternative approach will be followed in which $d_{s}$ is simply set equal to the cylindrical fibre diameter $D_{f}$.

The permeability prediction provided by the RUC model for through plane flow (Woudberg, 2017) is given by the following RUC TP model:

$B_{T P}=D_{f}^{2}\left[\frac{(1-\sqrt{\phi})^{3}}{13.5 \phi^{3 / 2}}\right]$.

The RUC TP model results from assigning twice a weight of $1 / 2$ to flow perpendicular to arrays of 1D fibrous media due to the two fibres in Fig. 1 that are both orientated in directions perpendicular to the streamwise direction (Woudberg, 2017). This was done in conjunction with the mixing rules of Mattern and Deen (2008) and also in analogy to the weighted average procedure in which 

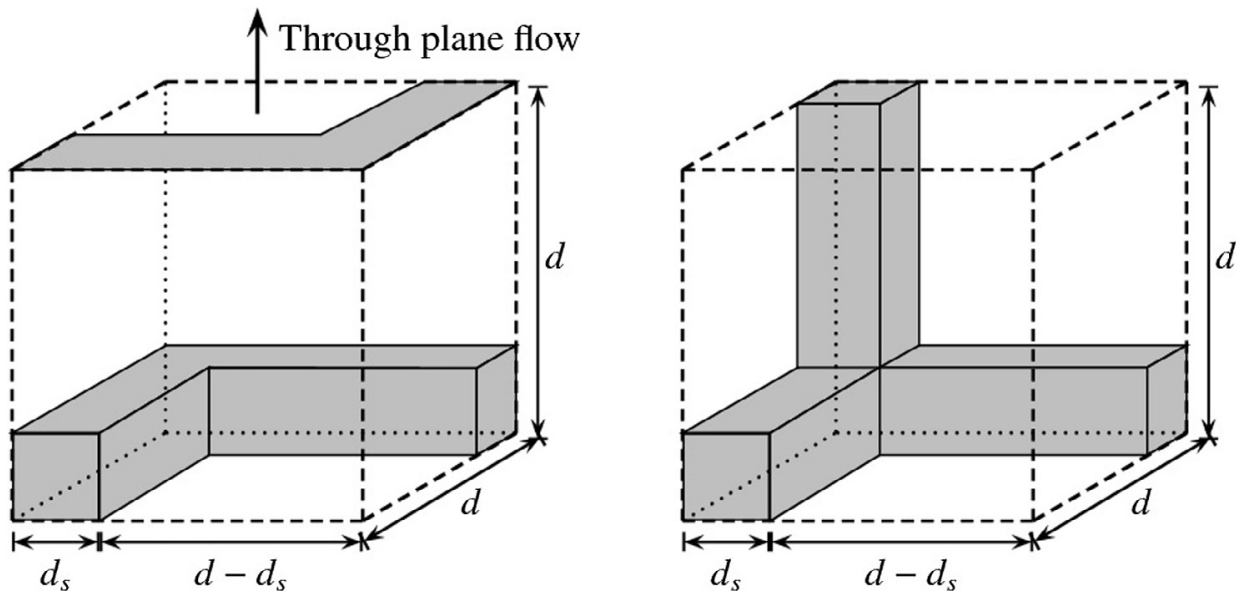

Fig. 1. RUC models for through plane flow (left) and flow through isotropic media (right) (Woudberg, 2017).

a weight of $1 / 3$ is assigned to the permeability prediction for flow parallel to $1 \mathrm{D}$ fibrous media and a weight of $2 / 3$ to that for flow perpendicular to $1 \mathrm{D}$ fibrous media in order to mathematically construct the isotropic model. As a result of equal weights being assigned in the RUC TP model, the permeability prediction is similar to that of flow perpendicular to $1 \mathrm{D}$ fibrous media.

The RUC model for flow through isotropic media is given by the following RUC iso model:

$B_{\text {iso }}=D_{f}^{2}\left[\frac{1}{3}\left[\frac{48 \phi^{2}}{(1-\phi)^{3}}\right]+\frac{2}{3}\left[\frac{13.5 \phi^{3 / 2}}{(1-\sqrt{\phi})^{3}}\right]\right]^{-1}$.

In Section 3 the three fibrous media are described that will be used to assess the models. The measured geometrical parameters will be provided and the methods that were used for the characterization will be discussed.

\section{Experimental data}

Table 1 contains details about the three fibrous filters that were used in this study for assessment of the models discussed in Sections 1 and 2. Two of the three media were characterized in the GEPEA laboratory and are used for particle removal from air: a raw filter of G4 type (named Medium A) according to the European Standard EN 779-2012 and the fibrous support of a bi-layered media of E10 type (named Medium B) according to the European Standard EN 1822. The experimental characterization of the bilayered medium, and of its fibrous support is detailed in Theron et al. (2017). The sample thicknesses $Z$ have been measured with a caliper for Medium A. For Medium B it was measured with more accuracy due to the facility developed by the LGP2 Laboratory (also used by Zerrouati et al., 2015) to measure the thickness of paper samples at low pressure. The fibre diameters $D_{f}$ have been determined by picture treatment of the SEM observations and the mean porosities $\varepsilon_{\text {mean }}$ have been obtained by application of mercury porosimetry. The experimental permeabilities $B_{\exp }$ have been determined with the aid of permeametry. Pressure drop measurements were carried out in a ventilated duct with a square cross section of $10 \times 10 \mathrm{~cm}^{2}$ in the $0.1-2.0 \mathrm{~m} \cdot \mathrm{s}^{-1}$ air velocity range. The permeability was derived from the linear part of the curve using the Darcy correlation:

$\frac{\Delta P}{Z}=\frac{\mu}{B_{\exp }} V$

where $\mu$ and $V$ respectively denote the air viscosity and the magnitude of the superficial velocity. For each medium the deviation in measured permeability is calculated on the basis of the sample thickness deviation.

The last filter (named Medium C) has been characterized and studied by Manzo et al. (2016) for the removal of liquid aerosol by coalescence filtration. It consists of five sheets of a medium made of stainless steel fibres, of $2.0 \mathrm{~mm}$ thickness. The porosity of the medium has been measured by a pycnometer method, and the permeability by a Frazier Permeability Tester.

Media $A$ and $C$ exhibit permeabilities of similar orders of magnitude despite their distinct characteristics. The porosity of Medium $C$ is higher than that of Medium A, and generally very high, therefore making it interesting to evaluate the ability of the models to predict the permeability. Medium B is less permeable than the other two media by one order of magnitude. The different media to be used in the assessment were thus carefully selected in order to investigate the model performances taking into account the different media characteristics.

In Section 4 the models discussed in Sections 1 and 2 are assessed against the experimental data provided in Table 1.

\section{Comparison between predicted and experimental permeabilities}

Fig. 2 compares the experimental permeability of Medium A to those predicted by the different models from the literature. Concerning the experimental permeability, the error bars reflect the deviation due to the measured thickness variation indicated in Table 1 . For the permeability predictions the error bars represent the higher permeability range obtained by taking into consideration the experimental deviation associated with the fibre diameter

Table 1

Properties of the studied fibrous media (data on the stainless steel medium obtained from Manzo et al. (2016)).

\begin{tabular}{|c|c|c|c|c|c|}
\hline Medium reference & Material & $Z(\mathrm{~mm})$ & $D_{f}(\mu \mathrm{m})$ & $\varepsilon_{\text {mean }}(-)$ & $B_{\exp }\left(\mathrm{m}^{2}\right)$ \\
\hline A & Cotton/polyester & $2.8 \pm 0.7$ & $13.8 \pm 4.4$ & $0.92 \pm 0.01$ & $2.02 \times 10^{-9} \pm 0.5 \times 10^{-9}$ \\
\hline B & Polyester & $0.918 \pm 0.022$ & $24.0 \pm 0.1$ & $0.85 \pm 0.01$ & $8.23 \times 10^{-10} \pm 0.2 \times 10^{-10}$ \\
\hline C & Stainless steel & 10.0 & $6.5 \pm 0.1$ & $0.986 \pm 0.007$ & $2.76 \times 10^{-9} \pm 0.003 \times 10^{-9}$ \\
\hline
\end{tabular}




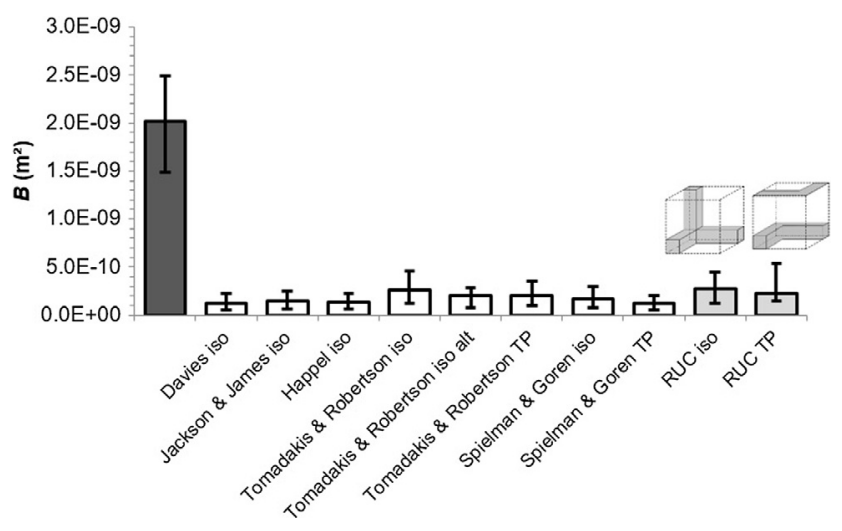

Fig. 2. Permeability predictions of various models from the literature compared to the experimental permeability of Medium A.

or porosity. This deviation is associated with the fibre diameter for Medium A, and with the porosity for Media B and C. It is evident from Fig. 2 that all the models significantly under-predict the experimental permeability. The two model predictions closest to the experimental permeability are those provided by the isotropic model of Tomadakis and Robertson and the isotropic RUC model which exhibit $B_{\exp }$ to $B_{\text {iso }}$ ratios of 7.7 and 7.3 , respectively. The RUC through plane model yields a ratio of 8.8 whilst for the other model predictions the ratio ranges between 14.2 and 16.7.

The same comparison is made for Medium B in Fig. 3. As for Medium A, all the models under-predict the permeability, but with lower $B_{\text {exp }}$ to $B_{\text {mod }}$ ratios. The two models providing the most accurate permeability predictions compared to the experimental value are again the isotropic model of Tomadakis and Robertson and the RUC iso model which yield $B_{\text {exp }}$ to $B_{\text {mod }}$ ratios of 4.5 and 4.1 , respectively. Contrary, the Spielman and Goren through plane model yields the maximum ratio of 7.9 .

Fig. 4 compares the experimental permeability of Medium C of Manzo et al. (2016) to those predicted by the different models from the literature. Also in this case the under-prediction is less than in the case of Medium A. The $B_{\exp }$ to $B_{\text {mod }}$ ratios obtained for the isotropic model of Tomadakis and Robertson and the RUC iso model are 1.2 and 1.6, respectively. The two other models of Tomadakis and Robertson also perform well, with $B_{\exp }$ to $B_{\bmod }$ ratios of 1.5 for both the isotropic alternative and the through plane versions. The maximum ratio of 7.7 is obtained with the model of Happel. In Fig. 4 the model deviations, represented by the error bars, are significant for the three models of Tomadakis and Robertson and the two RUC models although the experimental permeability falls

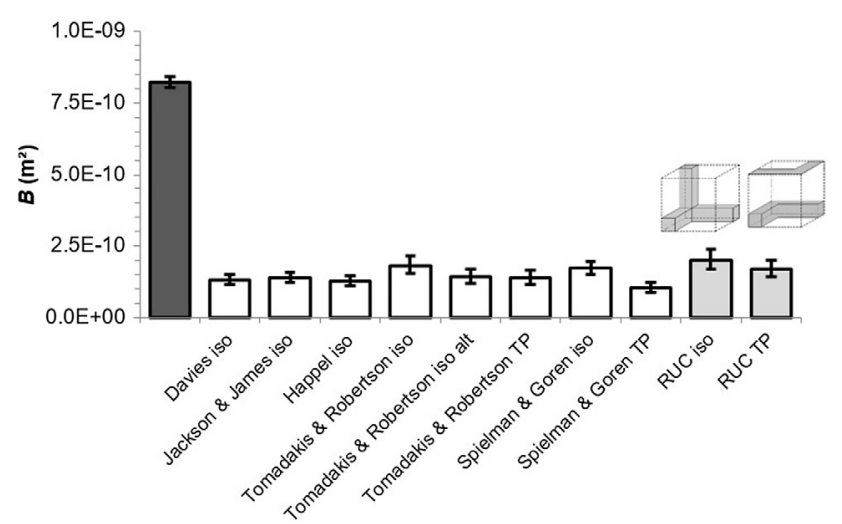

Fig. 3. Permeability predictions of various models from the literature compared to the experimental permeability of Medium B.

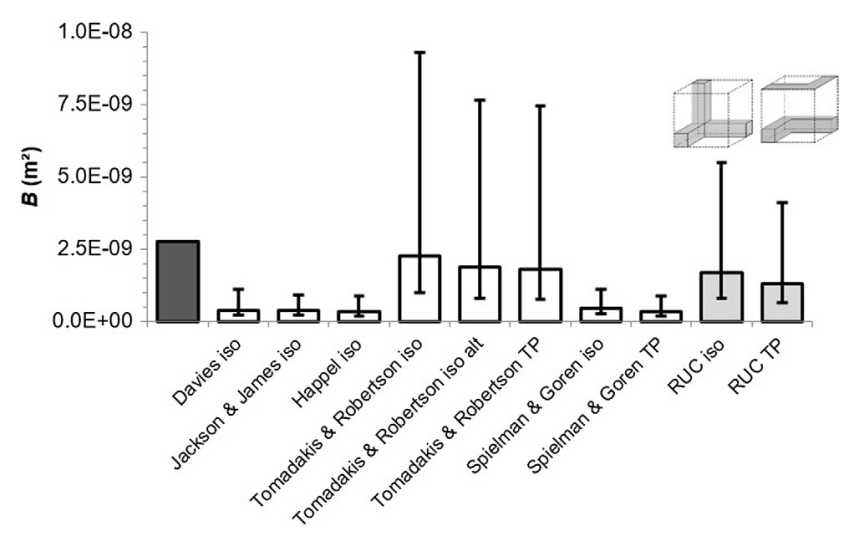

Fig. 4. Permeability predictions of various models from the literature compared to the experimental permeability of Medium C reported by Manzo et al. (2016).

within the deviation range of the predicted permeability. This may be due to the fact that the permeability models are very sensitive to the porosity at such high values. Fig. 5 depicts the sensitivity of the permeability to the porosity predicted by the five aforementioned models. In this figure the grey rectangle represents the range of porosity of Medium $C$ that is $98.6 \pm 0.7 \%$. It shows that (depending on the model considered) the experimental permeability of Medium $\mathrm{C}$ is reached by the models for porosity values ranging from 98.7 to $99.1 \%$.

Figs. $2-4$ show that in the range of permeability tested $(8.23 \times$ $10^{-10}-2.02 \times 10^{-9} \mathrm{~m}^{2}$ ), all the models under-predict the experimental permeability value. This phenomenon can be explained by the in-plane heterogeneous nature of fibrous media, which can be rather pronounced. Indeed, it is highly probable that the mean porosity does not reflect well the real porous structure of a fibrous medium. It can be assumed that the fibrous media exhibit local porosity variations in the plane of the fibre sheets, and especially some zones more porous than the mean porosity value which can lead to preferential air flow paths through the sheets.

Medium $A$ is a rather rough medium, which explains why it leads to the highest $B_{\text {exp }}$ to $B_{\text {mod }}$ ratio. Even if Medium $C$ has a similar permeability, which is the case, its lower $B_{\text {exp }}$ to $B_{\text {mod }}$ ratio can be due to its layered structure formed by the stacking of different sheets. Indeed, the potential local heterogeneities (resulting in local in-plane porosity variations) of each sheet may be averaged by the stacking of the five sheets, and thus may be of smaller magnitude than the local heterogeneities (resulting from medium roughness) exhibited by Medium A. Finally concerning Medium $\mathrm{B}$, its $B_{\exp }$ to $B_{\text {mod }}$ ratio lies between those obtained for Media A

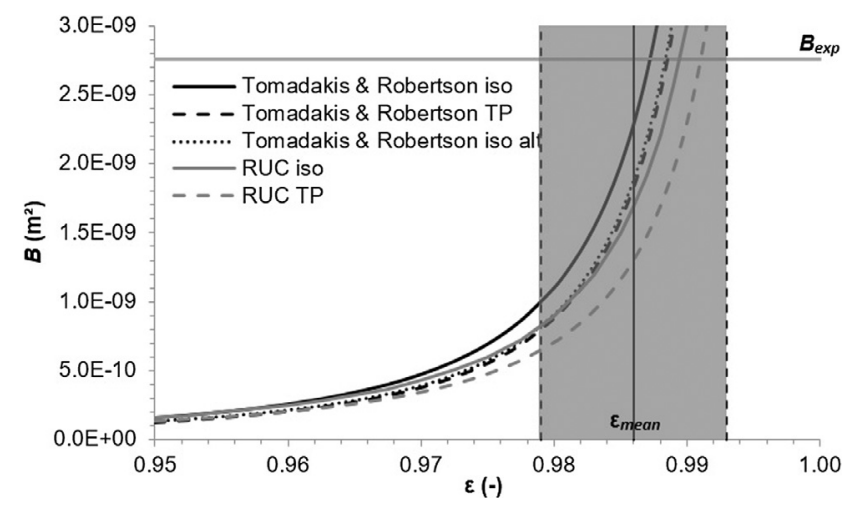

Fig. 5. Sensitivity of the permeability predicted by various models to the mean media porosity in the aim of predicting the permeability of Medium $C$ reported by Manzo et al. (2016). 
and $\mathrm{C}$. The structure of Medium B is less rough than that of Medium $A$, so its structural properties may be more homogeneous even at local scale. Medium B, on the other hand, is thinner than Medium $C$ which can lead to more preferential air flow paths in case of potential local heterogeneities.

The local variations of porosity cannot be quantified by methods such as mercury porosimetry and picnometry. These local variations should not be confused with the porosity deviations indicated in Table 1 that reflect the variations in terms of mean porosity obtained by repeating the measurement on different medium samples. To the knowledge of the authors, the only method that enables local porosity variations to be detected is X-ray micro-tomography. Such analyses have been performed on Medium B (e.g. Theron et al., 2017). The measurement performed on a sample of $10 \mathrm{~mm}$ in diameter enabled the determination of a similar mean porosity to that obtained by mercury porosimetry. It also enabled one to calculate the porosity of five noninterpenetrated circular zones distributed on the sample and to highlight an in-plane local porosity range. In order to be more representative, the same calculations are presented in Fig. 6 but on 13 circular zones of $1.49 \mathrm{~mm}$ in diameter, corresponding to local areas of $1.74 \mathrm{~mm}^{2}$. The average porosity of those zones is $85.6 \%$, which is in good agreement with the value of $85.5 \%$ obtained for the complete sample and the $85.0 \pm 1.0 \%$ obtained by mercury porosimetry. Separately these zones exhibit porosities ranging from 81.2 to $89.1 \%$, which indicate that the porous structure of this medium is rather heterogeneous.

Fig. 7 presents the sensitivity of the permeability of the tested Medium B to the porosity predicted by the two best fitting models. Those models are the isotropic Tomadakis and Robertson model and RUC iso model, as well as the through plane Tomadakis and Robertson model and the RUC TP model. In this figure the grey zone represents the range of porosity of this medium obtained by mercury porosimetry that is $85.0 \pm 1.0 \%$, and the vertical dashed bar represents the maximum value detected locally by X-ray micro-tomography (89.1\%). The two isotropic models fit better with the experimental values than their through plane counterparts and lead to the highest permeability values. The RUC models predict higher values than those predicted by the models of Tomadakis and Robertson. The RUC models thus appear rather satisfactory as they are purely analytical compared to the models of Tomadakis and Robertson which involve some empirical coefficients that have been determined on the basis of curve fitting of experimental data. Moreover, the anisotropic character of Medium B seems to have less influence on the permeability than its local

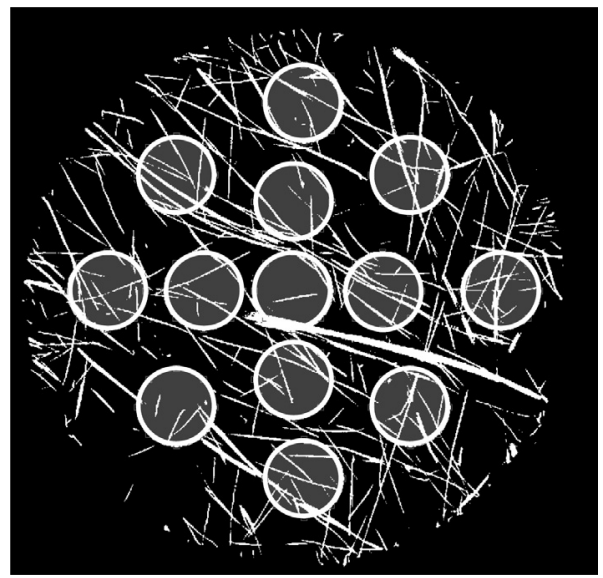

Fig. 6. Evaluation of in-plane variation in the porosity for Medium B obtained by micro-tomography on a $10 \mathrm{~mm}$ diameter sample.

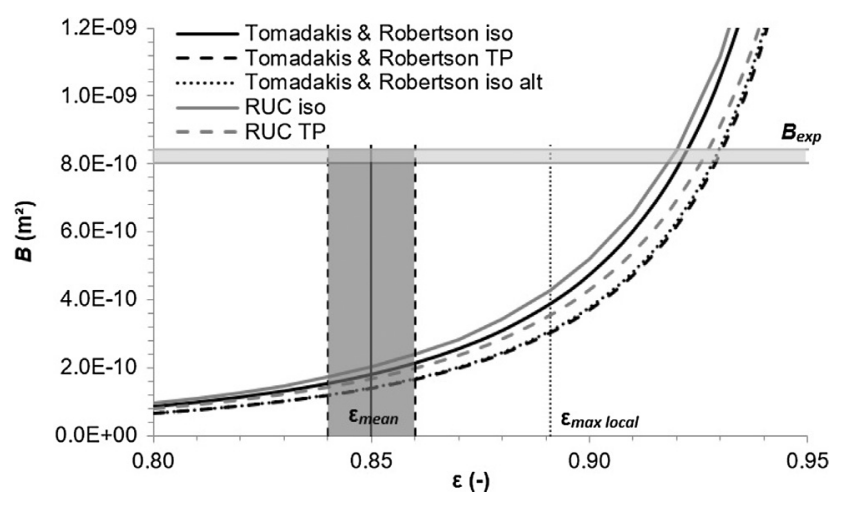

Fig. 7. Sensitivity of the permeability predicted by various models to the media porosity in the aim of predicting the permeability of Medium B.

heterogeneous porous structure (due to the in-plane porosity variations that it exhibits).

Indeed, Fig. 7 shows that in the porosity range between the mean value measured by mercury porosimetry (85\%) and the maximum local value detected by X-ray micro-tomography $(89.1 \%)$ the permeability increases by a factor of approximately 2.2 for the five models considered, whereas for each model the ratio of the permeability predicted by the isotropic version to the through plane one is approximately 1.3 .

For reference and completeness sake, Fig. 8 is included in which the RUC iso model for fibrous media is compared to the permeability prediction proposed by Hooman and Dukhan (2013) for foams, experimental data for foams by Mancin et al. (2010) and Dietrich et al. (2009) as well as the permeability predicted by the Ergun equation for granular packed beds. The solid filled symbols represent the experimental foam data of Mancin et al. (2010) and the non-filled symbols the foam data of Dietrich et al. (2009). The circles (filled and non-filled) represent the experimental data for foams, the squares represent the RUC iso model, the triangles Eq. (18a) of Hooman and Dukhan (2013) and the diamonds the permeability predicted by the Ergun equation (Ergun, 1952).

The foams studied by Mancin et al. (2010) have an average strut diameter of $443 \mu \mathrm{m}$ and porosities within the range $0.90 \leq \varepsilon \leq$ 0.96 and the Alumina foams of Dietrich et al. (2009) an average diameter of $537 \mu \mathrm{m}$ and a porosity range of $0.75 \leq \varepsilon \leq 0.86$. These average diameters are 1-2 orders of magnitude larger than those of

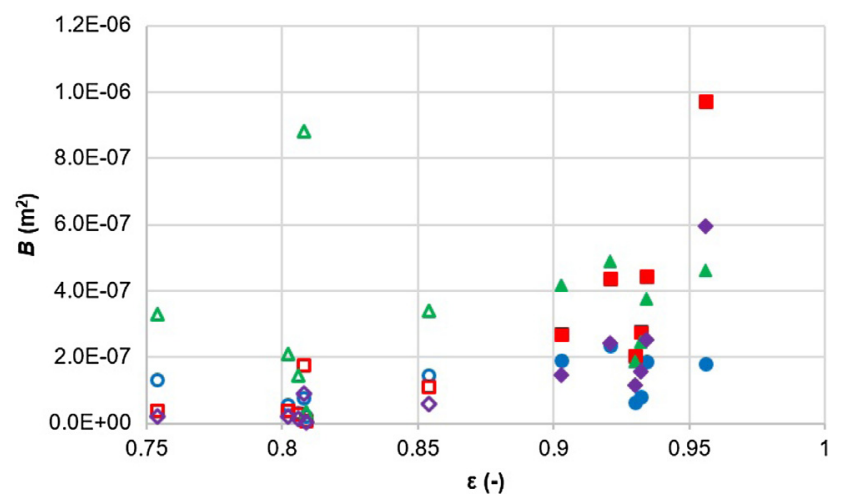

Fig. 8. Comparison of the permeability predicted by the RUC iso model for fibrous media with models and data from the literature for foams and granular media. Solid filled symbols are applicable to experimental foam data of Mancin et al. (2010) and non-filled symbols to foam data of Dietrich et al. (2009). Circles denote experimental data for foams, Squares represent the RUC iso model, Triangles Eq. (18a) of Hooman and Dukhan (2013) and Diamonds the permeability predicted by the Ergun equation. 
Media A, B and C for similar porosity values. This leads to permeability values of $2-3$ orders of magnitude larger than that of the fibrous filters considered in this study. The experimental permeability values of Media A, B and C are therefore not indicated in Fig. 7. For direct comparison, it was assumed that the granular particle diameters are equal to the fibre diameters and foam strut diameters. Under this assumption, good correspondence is obtained between the Ergun equation and the models for fibrous and foamlike media, although the range of porosities indicated falls beyond that of packed beds for which the Ergun equation is applicable. Although the permeability prediction of the RUC iso model corresponds well with the foam data and models for foams and granular media, it still under-predicts the permeability data for the fibrous media considered in this study. This is also true for the other fibre models discussed in Section 1. An investigation into the discrepancies between the model predictions and experimental data for fibrous media therefore deserves attention.

Up to this point, the fibre models presented only take into account the mean porosities and fibre diameters, in terms of input parameters to the models. The local variations of in-plane porosity could be an explanation for the under-prediction in permeability. In particular, the highest detected porosity value gives an indication of the preferential paths that may be followed by the air flow, although it does not represent the whole porosity field.

A sensitivity analysis is performed on the RUC iso model in order to illustrate the effect of the local variations of in-plane porosity on the permeability prediction. Let $\delta_{\min }$ represent the estimated percentage (expressed as a fraction between 0 and 1 ) of flow through local lower porosity regions (for which the maximum solid volume fraction $\phi_{\max }$ will be used as an upper bound). The fraction of flow through local higher porosity regions (for which the minimum solid volume fraction $\phi_{\min }$ will be used as a lower bound)) is calculated as $\delta_{\max }=1-\delta_{\min }$. Introducing the local minimum and maximum solid volume fractions into the permeability prediction into Eq. (10), leads to the following expression for the permeability prediction of the RUC iso sensitivity model (abbreviated, $B_{\text {sens }}$ ):

$$
\begin{aligned}
B_{\text {sens }}= & \delta_{\min } D_{f}^{2}\left[\frac{1}{3}\left[\frac{48 \phi_{\max }^{2}}{\left(1-\phi_{\max }\right)^{3}}\right]+\frac{2}{3}\left[\frac{13.5 \phi_{\max }^{3 / 2}}{\left(1-\sqrt{\phi_{\max }}\right)^{3}}\right]\right]^{-1} \\
& +\left(1-\delta_{\min }\right) D_{f}^{2}\left[\frac{1}{3}\left[\frac{48 \phi_{\min }^{2}}{\left(1-\phi_{\min }\right)^{3}}\right]+\frac{2}{3}\left[\frac{13.5 \phi_{\min }^{3 / 2}}{\left(1-\sqrt{\phi_{\min }}\right)^{3}}\right]\right]^{-1} .
\end{aligned}
$$

Fig. 9 shows the influence of the heterogeneity of Medium B on the model prediction provided by Eq. (12) by making use of the measured (by X-ray micro-tomography) $\phi_{\min }=1-\varepsilon_{\max }$ and $\phi_{\max }=1-\varepsilon_{\min }$ values for which $\varepsilon_{\min }=0.812$ and $\varepsilon_{\max }=0.891$.
As expected, the smaller $\delta_{\min }$, and thus the larger $\delta_{\max }$, the higher the permeability value. This implies that a greater percentage of fluid will flow through the local regions of $\phi_{\min }$ (corresponding to the local maximum porosity regions). The model prediction therefore tends to support that the fluid will flow mainly through local high porosity regions compared to local low porosity zones. Although the most accurate result is obtained when $\delta_{\text {min }}=0$ (as expected), the purpose of the sensitivity analysis, portrayed by Eq. (12), is to illustrate the significant effect that local porosity variations can have on the accuracy of the permeability prediction. The ratio of the permeability prediction for $\delta_{\min }=0$ to $\delta_{\min }=1$ is 3.8 .

For Medium B the local minimum and maximum porosity values used to assess this RUC iso sensitivity model comes from 13 values of local porosity (obtained on $1.74 \mathrm{~mm}^{2}$ zones). To be statistically more accurate, more local porosity values should be determined which would require additional tomography analyses on several medium samples. In general, the use of Eq. (12) is recommended in order to investigate the influence of the porosity variation when assessing a model prediction. Although Eq. (12) specifically involves the RUC iso model, the other permeability models can be expressed in a similar form, i.e.:

$B_{\text {sens }}=\delta_{\min } B_{\bmod }\left(\phi_{\max }\right)+\left(1-\delta_{\min }\right) B_{\bmod }\left(\phi_{\min }\right)$.

In the next section the RUC iso model will be adapted in order to better reflect on the stacking effect, i.e. the influence of anisotropy as a result of the stacking of fibrous medium sheets into layers constituting the real filter.

\section{Adaptations to RUC iso model}

Medium C studied by Manzo et al. (2016) were constructed by stacking layers of fibrous medium sheets and will therefore be considered in this section. Since the RUC iso model performs superior to the RUC TP model, the former model will be adapted to take the stacking effect into account.

The stacking of fibrous medium sheets into several layers creates an additional porosity as a result of the air spaces between the layers that needs to be incorporated into the model. Fig. 10 differs from the model on the right hand side of Fig. 1 in that the vertical fibre leg is shifted to the right in order to correctly portray the staggering in the streamwise direction of the piece-wise parallel plate geometry created by the indicated surfaces of the average fibre geometry adjacent to the RUC. All other model characteristics remain the same. The only other difference is the vertical cell dimension (parallel to the through plane flow direction) that is labelled $d_{t}$ in Fig. 10 which will now be allowed to take on values

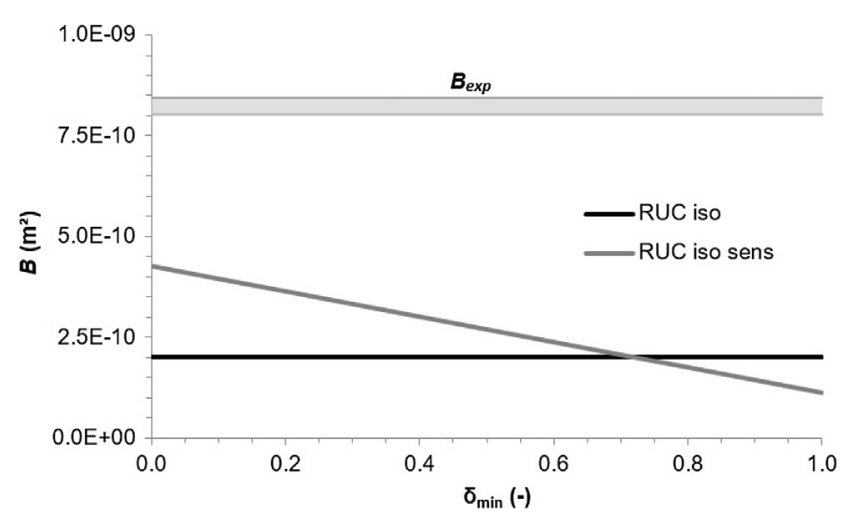

Fig. 9. Effect of fractions of local minimum and maximum porosity values on the permeability predicted by the RUC iso sensitivity model for Medium B.

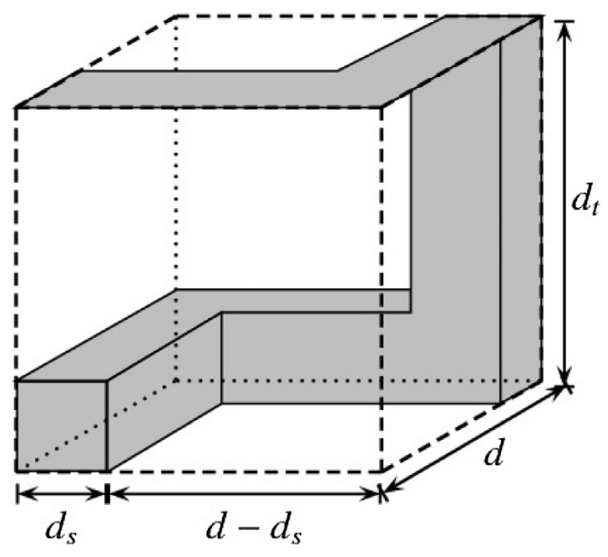

Fig. 10. Adapted RUC Iso model. 
that are larger than the in-plane cell dimension $d$. This implies that the open air space between the two solid sections, (representative of the stacking of fibrous medium sheets into layers) can be extended to take the additional porosity between medium layers into account. Although the model assumes that the medium sheets are isotropic, which is in good agreement with permeability results (see Fig. 4), the objective is to incorporate the stacking effect into the permeability prediction of the RUC iso model.

The dimension $d_{t}$ is allowed to be extended, such that $d_{t}=\gamma d$, with $\gamma(\geq 1)$ an anisotropy factor representing the factor by which the RUC cell dimension is extended to account for the additional porosity as a result of the stacking of sheets. The porosity affected by the adaptation, represented by $\varepsilon_{\text {adapt }}$, can accordingly be expressed as:

$\varepsilon_{\text {adapt }}=\frac{U_{f}}{U_{o}}=\frac{U_{o}-U_{s}}{U_{o}}=\frac{d_{t} d-d_{s}^{2}}{d_{t} d}=\frac{\gamma d^{2}-d_{s}^{2}}{\gamma d^{2}}=1-\frac{1}{\gamma}\left(\frac{d_{s}}{d}\right)^{2}$,

where $U_{o}$ is the total cell volume, $U_{f}$ is the fluid volume and $U_{s}$ the solid volume in the RUC. Eq. (10) itself remains unchanged, since there is no change in the shear stresses because the amount of solid volume remains the same. Recall that the adjacent solid surface indicated at the top part of the RUC in Fig. 10 does not contribute to the solid volume in the RUC. It merely illustrates the effect of an adjacent layer that constitutes the piece-wise parallel plate geometry on which the flow is based. The following relationship is obtained from the 1D fibre model (Woudberg, 2017):

$\frac{d_{s}}{d}=\sqrt{1-\varepsilon_{\exp }}=\sqrt{\phi_{\exp }}$,

where $\varepsilon_{\exp }$ (and thus $\phi_{\exp }$ ) is the experimentally measured average porosity (and thus solid volume fraction) value. Eq. (15) can be used to calculate the $d_{s} / d$ ratio from the measured mean porosity value.

Knowing this value, the adapted (or apparent) porosity value $\varepsilon_{\text {adapt }}$ can be determined by substituting Eq. (15) into Eq. (14) and expressing the result in terms of the solid volume fraction, i.e.:

$\phi_{\text {adapt }}=\frac{\phi_{\exp }}{\gamma^{a}}$

or in terms of porosity:

$\varepsilon_{\text {adapt }}=1-\frac{1}{\gamma^{a}}\left(1-\varepsilon_{\exp }\right)$,

with $a=1$. According to Eq. (14), $\varepsilon_{\text {adapt }}$ will increase (and thus $\phi_{\text {adapt }}$ will decrease) with an increase in the value of $\gamma$, as expected. The effect of $1 \leq \gamma \leq 2$ on the permeability prediction of the RUC iso adapt model is shown in Fig. 10 by replacing $\phi$ in Eq. (10) with $\phi_{\text {adapt }}$ (given by Eq. (16)). The range of $\gamma$-values investigated resembles a vertical RUC dimension ranging from the cubic dimension $(\gamma$ $=1$ ) to a rectangular dimension having a through plane height of twice the length of the in-plane dimension $(\gamma=2)$.

Fig. 11 illustrates the significant effect of the dependency between the experimental and adapted solid volume fraction values. Although it is assumed that $d_{t}$ changes linearly with $d$, (arbitrarily chosen) alternative relationships, i.e. $a=2$ and 0.5 are also indicated in Fig. 11. The $\gamma$-values that provide exact correspondence between model prediction and $B_{\exp }$ for each $a$-value, i.e. $a$ $=2, a=1$ and $a=0.5$ are $\gamma=1.15, \gamma=1.30$ and $\gamma=1.75$, respectively. This illustrates the effect of the $a$-dependency on the anisotropic $\gamma$ factor. Each of the three corresponding $(a, \gamma)$-values yields a value of 0.989 for $\varepsilon_{\text {adapt }}$. This porosity value corresponds to the one at which the RUC iso model fits the experimental permeability value (in Fig. 5), which is to be expected. It must be noticed that this porosity value also falls within the deviation range of the mean fibrous medium porosity measured by pycnometry $(0.986 \pm$ 0.007 ). With $\varepsilon_{\text {adapt }}=0.989$ being close to the value of the measured

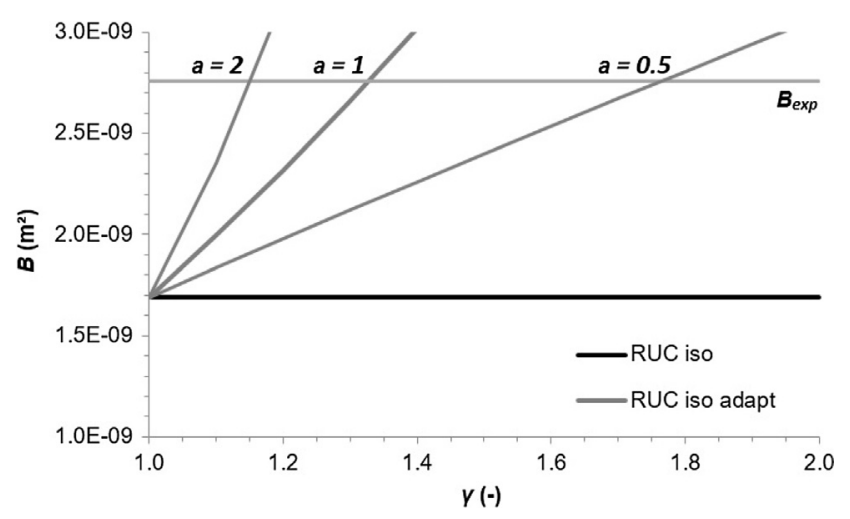

Fig. 11. Effect of both value and formulation of the anisotropic factor $\gamma$ on the permeability predicted by the RUC iso model for Medium C reported by Manzo et al. (2016).

mean porosity of a single fibrous sheet, tends to indicate that the stacking does not affect the filter apparent porosity significantly. However, whilst the measured mean porosity is used in the RUC iso model in order to predict the permeability, the RUC iso adapt model could be applied to calculate the porosity value to be used in the model prediction (i.e. $\varepsilon_{\text {adapt }}$ ) more accurately. It should be noted that although the permeability is sensitive to the $\gamma$ and $a$ values, as shown in Fig. 11, the $\varepsilon_{\text {adapt }}$ value is not. This is due to the high average porosity value of the fibrous medium considered. Further analysis should be conducted on lower porosity materials in order to establish the sensitivity of the calculated $\varepsilon_{\text {adapt }}$ value on the $\gamma$ and $a$ values.

Alternatively, the tortuosity could be used to determine $\varepsilon_{\text {adapt }}$, should such values be known. The geometrical tortuosity $\chi$ can be expressed in RUC notation as

$\chi=\frac{d_{e}}{d_{t}}=\frac{U_{f}}{A_{p \|} d_{t}}=\frac{\varepsilon_{\text {adapt }} U_{o}}{A_{p \|} d_{t}}=\frac{\varepsilon_{\text {adapt }} d^{2} d_{t}}{A_{p \| \mid} d_{t}}=\frac{\varepsilon_{\text {adapt }} d^{2}}{A_{p \|}}=\frac{\varepsilon_{\text {adapt }} d^{2}}{\left(d-d_{s}\right)^{2}}$,

where $d_{e}$ denotes the average length of the flow path in the RUC and $A_{p \|}$ is the streamwise cross-sectional flow area in the RUC (Woudberg, 2017). From Fig. 10 it can be deduced that $A_{p \| \mid}=(d-$ $\left.d_{s}\right)^{2}$. Substituting Eq. (15) into Eq. (18) leads to

$\varepsilon_{\text {adapt }}=\chi\left(1-\frac{d_{s}}{d}\right)^{2}=\chi\left(1-\sqrt{1-\varepsilon_{\text {exp }}}\right)^{2}$

The anisotropic factor $\gamma$ does not appear in Eq. (19) since it cancels out. The tortuosity factor appears instead and usually takes on a similar range of values as $\gamma$ (i.e. $1 \leq \chi \leq 2$ ). It may, however, be easier to obtain the value of $\gamma$ by measuring the average extended dimension between the stacking of fibre sheets than to determine tortuosity values, which gives preference to the use of Eq. (17).

\section{Conclusions}

Permeability values ranging from $8.23 \times 10^{-10}$ to $2.02 \times 10^{-9}$ $\mathrm{m}^{2}$ of three real fibrous media used for air filtration were compared to those predicted by numerous empirical and analytical models from the literature. The three media exhibit mean porosity and fibre diameter values of $85-99 \%$ and 6-24 $\mu \mathrm{m}$, respectively. Special attention is given to the analytical RUC model. It has been demonstrated that all the models under-predict the experimental permeability value, but with different orders of magnitude depending on the medium characteristics. Indeed the rougher the medium, which can be reflected by a significant in-plane heterogeneous nature, the higher the $B_{\text {exp }}$ to $B_{\text {mod }}$ ratio. 
Moreover it was found that for the media tested the best prediction is always reached by the Tomadakis and Robertson and the RUC models. The RUC model thus appears rather satisfactory as it is purely analytical compared to the model of Tomadakis and Robertson which involves some empirical coefficients. The isotropic versions of both models better predict the experimental values than their through plane counterparts. This indicates that the anisotropic character of the medium seems to have less influence on the permeability than its heterogeneous nature (due to the inplane porosity variations that it exhibits, for example).

It has also been illustrated that the mean porosity value (or solid volume fraction, typically measured by porosimetry or picnometry) used in the model predictions for the permeability is not adequate, especially if the heterogeneous nature is due to significant local in-plane porosity variations. Data of such local porosity variations were experimentally obtained for Medium B. A sensitivity analysis applied to the RUC iso model reveals that minimum or maximum solid volume fraction values incorporated into the model leads to a significant relative permeability deviation. An equation was developed to investigate the influence of the local porosity variation when assessing a model prediction, which is recommended to be tested in case of available local porosity data (obtained by X-ray micro-tomography for example).

Finally the stacking effect of fibrous sheets has been investigated. It has been shown that an adaptation of the RUC iso model by extending the RUC height in the through plane direction (representative of the increase of apparent porosity due to stacking) results in fitting the experimental permeability. This RUC iso adapt model allows one to calculate the value of the apparent porosity of the stacking to be used in the model. In the future it would require further analysis especially on lower porosity materials.

The results obtained in this study provide more insight into some of the physical phenomena that influence the permeability prediction of fibrous materials and contribute to the understanding of the significant under-prediction of the numerous models from the literature when compared to the experimental data. The investigations were focused on fibrous media used for air filtration, with their own structural properties, but could be also tested for other types of non-woven materials (such as papers, composites, textiles, etc.).

\section{Acknowledgements}

The authors gratefully acknowledge the Lydall company for supplying the fibrous support of a composite medium. The authors would also like to thank the LGP2 Laboratory (Laboratoire de Génie des Procédés Papetiers, Grenoble, France) for the thickness measurements, and the GEM Laboratory (Institut de Recherche en Génie Civil et Mécanique, Nantes, France) for the acquisition of micro-tomography data.

\section{References}

Clague, D.S. Phillips, R.J., 1997. A numerical calculation of the hydraulic permeability of three-dimensional disordered fibrous media. Phys. Fluids 9 $1562-1572$.

Davies, C.N., 1952. The separation of airborne dust and particles. Proc. Inst. Mech Eng. (Lond.) B1, 185-213.

Dietrich, B., Schabel, W., Kind, M., Martin, H., 2009. Pressure drop measurements of ceramic sponges - determining the hydraulic diameter. Chem. Eng. Sci. 64 3633-3640.

Drummond, J.E., Tahir, M.I., 1984. Laminar viscous flow through regular arrays of parallel solid cylinders. Int. J. Multiph. Flow 10 (5), 515-540.

Ergun, S., 1952. Fluid flow through packed columns. Chem. Eng. Prog. 48 (2), 89-94.

Gebart, B.R., 1992. Permeability of unidirectional reinforcements for RTM. J. Compos. Mater. 26 (8), 1100-1133.

Gervais, P.C., Bardin-Monnier, N., Thomas, D., 2012. Permeability prediction of fibrous media with bimodal fibre size distribution. Chem. Eng. Sci. 73, 239-248.

Happel, J., 1959. Viscous flow relative to arrays of cylinders. Am. Inst. Chem. Eng. J. 5 (2), 174-177.

Hooman, K., Dukhan, N., 2013. A theoretical model with experimental verification to predict hydrodynamics of foams. Transp. Porous Med. 100, 393-406.

Huang, X., Wang, Q., Zhou, W., Deng, D., Zhao, Y., Wen, D., Li, J., 2015. Morphology and transport properties of fibrous porous media. Powder Technol. 283, 618626.

Jackson, G.W., James, D.F., 1986. The permeability of fibrous porous media. Can. J. Chem. Eng. 64 (3), 364-374.

Jaganathan, S., Vadehi Tafreshi, H., Pourdeyhimi, B., 2008. A realistic approach for modelling permeability of fibrous media: 3-D imaging coupled with CFD simulation. Chem. Eng. Sci. 63, 244-252.

Mancin, S., Zilio, C., Cavallini, A., Rossetto, L., 2010. Pressure drop during air flow in aluminum foams. Int. J. Heat Mass Transf. 53, 3121-3130.

Manzo, G.M., Wu, Y., Chase, G.G., Goux, A., 2016. Comparison of nonwoven glass and stainless steel microfibre media in aerosol coalescence filtration. Sep. Purif. Technol. 162, 14-19.

Mattern, K.J., Deen, W.M., 2008. "Mixing rules" for estimating the hydraulic permeability of fiber mixtures. AIChE J. 54, 32-41.

Sangani, A.S., Acrivos, A., 1982. Slow flow past periodic arrays of cylinders with application to heat transfer. Int. J. Multiph. Flow 8, 193-206.

Soltani, P., Zarrebini, M., Laghaei, R., Hassanpour, A., 2017. Prediction of permeability of realistic and virtual layered nonwovens using combined application of X-ray $\mu \mathrm{CT}$ and computer simulation. Chem. Eng. Res. Des. 124, 299-312 (accepted for publication).

Spielman, L., Goren, S.L., 1968. Model for predicting pressure drop and filtration efficiency in fibrous media. Environ. Sci. Technol. 2 (4), 279-287.

Tamayol, A., Bahrami, M., 2010. Parallel flow through ordered fibres: an analytical approach. J. Fluids Eng. 132, 1-7.

Tamayol, A., Bahrami, M., 2011. Transverse permeability of fibrous porous media Phys. Rev. E 83, 1-9.

Theron, F., Lys, E., Joubert, A., Bertrand, F., Le Coq, L., 2017. Characterization of the porous structure of a non-woven fibrous medium for air filtration at local and global scales using porosimetry and X-ray micro-tomography. Powder Technol. 320, 295-303.

Tomadakis, M.M., Robertson, T.J., 2005. Viscous permeability of random fiber structures: comparison of electrical and diffusional estimates with experimental and analytical results. J. Compos. Mater. 9 (2), 163-187.

Van Doormal, M.A., Pharoah, J.G., 2009. Determination of permeability of fibrous porous media using the lattice Boltzman method with application to PEM fue cells. J. Numer. Methods Fluids 59, 75-89.

Woudberg, S., 2017. Permeability prediction of an analytical pore-scale model for layered and isotropic fibrous porous media. Chem. Eng. Sci. 164, 232-245.

Zerrouati, A., Rueff, M., Bouchekima, B., 2015. Study of paper transverse shrinkage during thermal drying. Dry. Technol. 331 (10), 1170-1179. 